\title{
Evaluation of Zinc and Homocysteine Status in Pregnant Women and Their Association with Pre-eclampsia in Jordan
}

\author{
Nawal Ahmad Al-Sakarneh ${ }^{1,2}$ and Rima Hussein Mashal ${ }^{2}$ \\ ${ }^{1}$ Food Physical Laboratory Division, Administration Laboratories Directoraite, Jordan Food and Drug Administration (JFDA), \\ Amman 11942, Jordan \\ ${ }^{2}$ Department of Nutrition and Food Technology, Faculty of Agriculture, The University of Jordan, Amman 11942, Jordan
}

\begin{abstract}
Pre-eclampsia (PE) is considered a major complication of pregnancy. Hyperhomocyteinemia (H-Hcy) has been proposed to be associated with a number of placenta-mediated diseases, such as PE. Zinc ( $\mathrm{Zn}$ ) is involved in the regulation of total homocysteine (Hcy) levels. A case-control study design was used to examine serum Zn and Hcy statuses, and their association to PE risk. Thirty pregnant women with PE $21 \sim 35$ years of age, and 30 matched healthy pregnant women were recruited from Amman, Jordan. Plasma Hcy was measured using liquid chromatography-mass spectrometry, and $\mathrm{Zn}$ was measured using atomic absorption. Hcy levels were significantly higher among women with PE compared with controls $(16.35 \pm 0.43$ and $7.25 \pm 0.21 \mu \mathrm{mol} / \mathrm{L}$, respectively; $P<0.05)$. However, there was no significant difference in $\mathrm{Zn}$ levels between women with PE and controls $(65.37 \pm 1.27$ and $63.71 \pm 1.24 \mu \mathrm{g} / \mathrm{dL}$, respectively; $P>0.05)$. Blood levels of Hcy $(\mu \mathrm{mol} / \mathrm{L})$ were positively associated with systolic and diastolic blood pressure $(\beta=3.54$ and $\beta=1.81$, respectively; $P<$ $0.05)$, and $\mathrm{Zn}$ levels [odds ratios $(\mathrm{OR})=0.84 ; 95 \%$ confidence intervals $(\mathrm{CI}): 0.71 \sim 0.98$ ] were significantly associated with PE risk $(P<0.05)$. Although women with PE had significantly higher Hcy levels than controls, H-Hcy was not associated with increased PE risk. However, there was a strong association between severity of hypertension and serum Hcy levels, and serum $\mathrm{Zn}$ levels were inversely associated with H-Hcy. The likelihood of PE was significantly higher in women who were Zn deficient compared with healthy controls. To conclude, early management of H-Hcy and associated risk factors may be effective in decreasing the incidence of PE.
\end{abstract}

Keywords: hyperhomocysteinemia, pregnancy and pre-eclampsia, total homocysteine, zinc

\section{INTRODUCTION}

Pregnancy and its complications are responsible for about 600,000 deaths worldwide every year, half of which are due to risky pregnancies (Vafaei et al., 2015). Pre-eclampsia (PE) is considered a major cause of maternal morbidity and mortality, and contributes to intrauterine growth restriction, preterm delivery, and prenatal mortality (Harma et al., 2005). Women with pregnancy-induced hypertensive disorders may progress from mild disease to a more serious condition. Types of pregnancy-induced hypertension include hypertension fluid retention mild PE, severe PE, and eclampsia (WHO, 2017). The exact mechanism underlying the etiology of PE remains unclear (Khosrowbeygi and Ahmadvand, 2011), however, the incidence is higher in developing countries than developed countries (Ghulmiyyah and Sibai, 2012).
Although global estimates of some micronutrients deficiencies are not available, population studies have shown that the percentage of pregnant women with zinc $(\mathrm{Zn})$ deficiency ranges from $15 \sim 74 \%$ in South Asia (Gernand et al., 2016). Micronutrient deficiencies are usually identified in pregnant women, and trace element deficiency may increase susceptibility to developing PE (Akinloye et al., 2010). Some of these trace elements can regulate the balance between free radicals and antioxidants (Roberts et al., 2003). Total homocysteine (Hcy) is a sulfur containing amino acid involved in processes such as lipid peroxidation and oxidative stress. Hcy is metabolized through transsulfuration and remethylation pathways (Khosrowbeygi and Ahmadvand, 2011). Hyperhomocysteinemia (H-Hcy) is a risk factor for cardiovascular diseases and common obstetric problems (Tug et al., 2003). Moreover, some studies have indicated that decreased 
concentrations of $\mathrm{Zn}$ is associated with fetal malformation, fetus growth restriction, preterm delivery, $\mathrm{PE}$, and bleeding after delivery (Rafeeinia et al., 2014). H-Hcy is considered a risk factor for endothelial dysfunction and vascular diseases, such as atherosclerosis. It has been hypothesized that maternal H-Hcy is associated with several placenta-mediated diseases such as PE (Khosrowbeygi and Ahmadvand, 2011). Furthermore, $\mathrm{Zn}$ is believed to be involved in regulating Hcy levels via mediating methionine synthease and betainehomocysteine methyl transferase, both of which are Zn-dependent metallo-enzymes (Jing et al., 2014). Therefore, it may be hypothesized that Hcy and $\mathrm{Zn}$ status are associated with the risk of PE.

$\mathrm{PE}$ is considered a major complication of pregnancy, but little is known about its etiology. Many theories about its etiology and pathogenesis have been proposed, including endothelial dysfunction (Rafeeinia et al., 2014). However, limited data are available on plasma Hcy status during pregnancy and on the occurrence of H-Hcy in women with PE in different geographical areas. To date, studies on the impact of $\mathrm{Zn}$ status on Hcy levels in women with PE are limited, particularly in Jordan. Therefore, the aim of the present study was to examine the associations between serum levels of $\mathrm{Zn}$ and Hcy and risk of PE in a selected sample of healthy, PE Jordanian women.

\section{MATERIALS AND METHODS}

\section{Study design and subjects}

A case-control study was conducted from December 2015 to April 2016 in Amman, Jordan. Thirty pregnant women with known PE were matched with 30 healthy pregnant women by maternal and gestational age. All women were recruited from the Obstetrics and Gynecology Department, Al-Bashir Hospital and Al-Hussein Medical Center. Eligible participants were pregnant women $21 \sim$ 35 years of age with PE at 20 40 weeks gestational age. Participants were excluded from the study if they met one of the following conditions: smoker, multiple gestation, diabetes mellitus, chronic hypertension, heart failure, renal disease, inflammatory or infective disorders, and infectious disease. A signed consent form was obtained from all subjects who were eligible to participate in this study. The protocol was approved by the Protection of Human Subjects Ethics Committee (No. 1429/3, Amman, Jordan) of the school of graduate studies at the University of Jordan, in accordance with the ethical guidelines described in the Declaration of Helsinki. All data were collected, retrived, and reported in a confidential manner.

\section{Definition of selected variables}

Information on selected factors that might influence nu- trition during pregnancy, including reproductive history such as age, gestational age, parity, and family history of PE, were obtained. Parity was defined as "yes" if the mother had experienced one or more pregnancies and "no" if she has never previously experienced pregnancy. Additional information on use of supplemental vitamins was obtained for all participants. Supplement use was defined as "yes" if the mother was a supplement user and "no" if the mother was not a supplement user. Anthropometric measurements including weight and height were performed. Body mass index (BMI) before and during pregnancy were calculated: BMI (1) was BMI before pregnancy and BMI (2) was BMI during pregnancy. Systolic and diastolic blood pressure was obtained for all participants from their medical records.

\section{Evaluation of biochemical predictors}

Blood samples were obtained from all participants. Samples were treated with ethylene diamine tetra acetic acid and separated on gels to determine serum total Hcy and $\mathrm{Zn}$ levels. Total Hcy was defined as the sum of all Hcy species in plasma/serum, including free and proteinbound forms.

Blood samples were transported in an ice box to a private laboratory for analysis. Serum was obtained by centrifuging whole blood samples for $10 \mathrm{~min}$ at $4^{\circ} \mathrm{C}$ within half an hour of collection. Blood samples were stored at $-80^{\circ} \mathrm{C}$ until they were analyzed for serum Hcy and $\mathrm{Zn}$. Serum Hcy was measured using liquid chromatographymass spectrometer (LCMS-8030, Shimadzu Corporation, Kyoto, Japan), and $\mathrm{Zn}$ levels were measured using Atomic Absorption (Shimadzu AA-6300, Shimadzu Corporation). Pregnant women with $\mathrm{Zn}$ levels of $\leq 65(\mu \mathrm{g} / \mathrm{dL})$ were considered $\mathrm{Zn}$ deficient and those with $\mathrm{Zn}$ levels $\mathrm{Z}$ 65 ( $\mu \mathrm{g} / \mathrm{dL}$ ) were considered Zn sufficient (Akhtar, 2013).

\section{Statistical analyses}

All statistical analyses were performed using IBM $^{\mathbb{R}}$ SPSS $^{\circledR}$ Statistics for Windows, version 19.0 (IBM Corp., Armonk, NY, USA). Means and standard error of the mean (SEM) were calculated for continuous variables, whereas categorical variables were reported as counts and frequency distribution (\%). Shapiro Wilk tests were used to test normality for all predictors including serum levels of $\mathrm{Zn}$ and Hcy. Therefore, based on normality tests, the group differences in PE risk factors were examined using parametric and non-parametric analyses, as appropriate. Differences between groups were estimated using analysis of variance (ANOVA) or Chi-square $\left(\chi^{2}\right)$, as appropriate. To examine the association between serum Hcy levels (dependent) and other indicators (independent), linear regression models were performed. Logistic regression analyses were performed to examine associations between serum Hcy levels, $\mathrm{Zn}$ levels, and the risk of PE. The rela- 
Table 1. Anthropometric and clinical characteristics of the sample population

\begin{tabular}{|c|c|c|c|}
\hline Variables & Cases $(n=30)$ & Controls $(n=30)$ & $P$-value \\
\hline Age (years) & $\begin{array}{c}28.3 \pm 0.8 \\
(21.0 \sim 35.0)\end{array}$ & $\begin{array}{c}28.0 \pm 0.9 \\
(21.0 \sim 35.0)\end{array}$ & 0.75 \\
\hline Gestational age (weeks) & $\begin{array}{c}33.1 \pm 0.8 \\
(23.0 \sim 38.0)\end{array}$ & $\begin{array}{c}31.7 \pm 0.9 \\
(23.0 \sim 38.0)\end{array}$ & 0.23 \\
\hline BMI (1) $\left(\mathrm{kg} / \mathrm{m}^{2}\right)$ & $\begin{array}{c}28.5 \pm 1.0 \\
(18.4 \sim 41.9)\end{array}$ & $\begin{array}{c}26.6 \pm 1.0 \\
(15.6 \sim 37.5)\end{array}$ & 0.18 \\
\hline BMI (2) $\left(\mathrm{kg} / \mathrm{m}^{2}\right)$ & $\begin{array}{c}34.4 \pm 1.1^{\mathrm{a}} \\
(25.0 \sim 47.0)\end{array}$ & $\begin{array}{c}30.8 \pm 1.0^{b} \\
(18.7 \sim 45.6)\end{array}$ & 0.02 \\
\hline Systolic blood pressure (mmHg) & $\begin{array}{c}154.2 \pm 2.6^{\mathrm{a}} \\
(140.0 \sim 180.0)\end{array}$ & $\begin{array}{c}114.2 \pm 1.4^{b} \\
(100.0 \sim 125.0)\end{array}$ & 0.00 \\
\hline Diastolic blood pressure $(\mathrm{mmHg})$ & $\begin{array}{c}95.2 \pm 1.1^{a} \\
(90.0 \sim 110.0)\end{array}$ & $\begin{array}{c}75.3 \pm 0.9^{b} \\
(70.0 \sim 80.0)\end{array}$ & 0.00 \\
\hline Parity ${ }^{1)}$ & $13(43.3)^{a}$ & $6(20)^{b}$ & 0.04 \\
\hline Family history ${ }^{1)}$ & $11(36.7)^{a}$ & $0(0)^{b}$ & 0.00 \\
\hline Supplement use (yes/no) $)^{1 / 2)}$ & $15(50)$ & $22(73.3)$ & 0.05 \\
\hline
\end{tabular}

Continuous variable are presented as mean \pm SEM with the range in parentheses.

Different letters $(a, b)$ indicated statistically significant difference at $P<0.05$.

BMI (1), body mass index before pregnancy; BMI (2), body mass index during pregnancy.

${ }^{1)}$ Categorical variables are presented as the number and percentage (\%) in each group.

${ }^{2}$ Supplement use was coded as "yes" if the mother was a supplement user and "no" if the mother was not a supplement user.

tive risks were reported as odd ratios (OR) with $95 \%$ confidence intervals (CI). $P$-values of $<0.05$ were considered significant.

\section{RESULTS}

The general anthropometric and selected clinical characteristics of the study subjects are shown in Table 1 . There were no significant differences $(P>0.05)$ between cases and controls in terms of age (years), gestational age (weeks), BMI before pregnancy $\left(\mathrm{kg} / \mathrm{m}^{2}\right)$, and supplement use during pregnancy. However, BMI during pregnancy was significantly higher in women with PE compared with controls $(34.35 \pm 1.09$ vs $30.81 \pm 1.04$, respectively, $P<0.05)$. Approximately $43.3 \%$ of women with PE and $20 \%$ of controls had carried pregnancies to a viable gestational age (Parity) $(P>0.05)$. The number of women with a family history of PE was significantly higher in those with PE compared with controls. Indeed, none of the healthy pregnant women had documented a family history of PE $(P<0.05)$. Furthermore, systolic and diastolic blood pressure was significantly higher in women with PE than in controls $(154.16 \pm 2.57$ and $114.16 \pm 1.44$ $\mathrm{mmHg}$ vs $95.16 \pm 1.05$ and $75.33 \pm 0.89 \mathrm{mmHg}$, respectively, $P<0.001)$.

The $P$-value for normality testing using Shapiro-Wilk tests for plasma Hcy and Zn levels was $P<0.05$ for both groups. We concluded that $\mathrm{Zn}$ and Hcy levels for this subset of subjects are not normally distributed. Mannmean differences in $\mathrm{Zn}$ and Hcy levels among groups are presented in Fig. 1. Serum levels of Hcy $(\mu \mathrm{mol} / \mathrm{L})$ was significantly higher in women with PE compared with controls $(16.35 \pm 0.43$ vs $7.25 \pm 0.21$, respectively; $P<$ $0.001)$. Although the mean concentration of $\mathrm{Zn}(\mu \mathrm{g} / \mathrm{dL})$ in controls was higher than in women with PE, the difference was not statistically significant $(65.37 \pm 1.27$ vs $63.71 \pm 1.24$, respectively; $P<0.05$ ).

\section{Association between serum Hcy levels and selected predictors}

Linear regression analyses were performed to assess the association between serum Hcy levels $(\mu \mathrm{mol} / \mathrm{L})$ and selected predicators in women with PE, controls, and the full cohort. In these analyses, the selected predicators were introduced as independent variables and serum Hcy as the dependent variable. The changes in Hcy levels $(\mu \mathrm{mol} / \mathrm{L})$ per unit change of the selected predicators are presented as $\beta$-coefficient with corresponding $P$-values (Table 2). Hcy levels were significantly associated with plasma $\mathrm{Zn}$ levels in pregnant women with PE but not in controls or in the full cohort $(\beta=0.18, P>0.05)$. None of the predictors were associated with serum Hcy levels $(\mu \mathrm{mol} / \mathrm{L})$ within the control group.

BMI before and during pregnancy was significantly associated with Hcy levels $(P<0.05)$. BMI of all women before pregnancy was associated with $0.25 \mu \mathrm{mol} / \mathrm{L}$ increases in Hcy levels $(P<0.05)$ in the full cohort. After adjusting for BMI before pregnancy and family history of PE, BMI during pregnancy was positively and significantly associated with $0.22 \mu \mathrm{mol} / \mathrm{L}$ increases in Hcy levels $(P<$ 0.05 ) in the full cohort. Both parity and family history of PE were strongly associated with Hcy levels in the full cohort ( $\beta=3.29$ and $\beta=4.61$, respectively, $P<0.05)$. To assess the association between serum $\mathrm{Zn}$ status and selected predictors, linear regression analyses were per- 

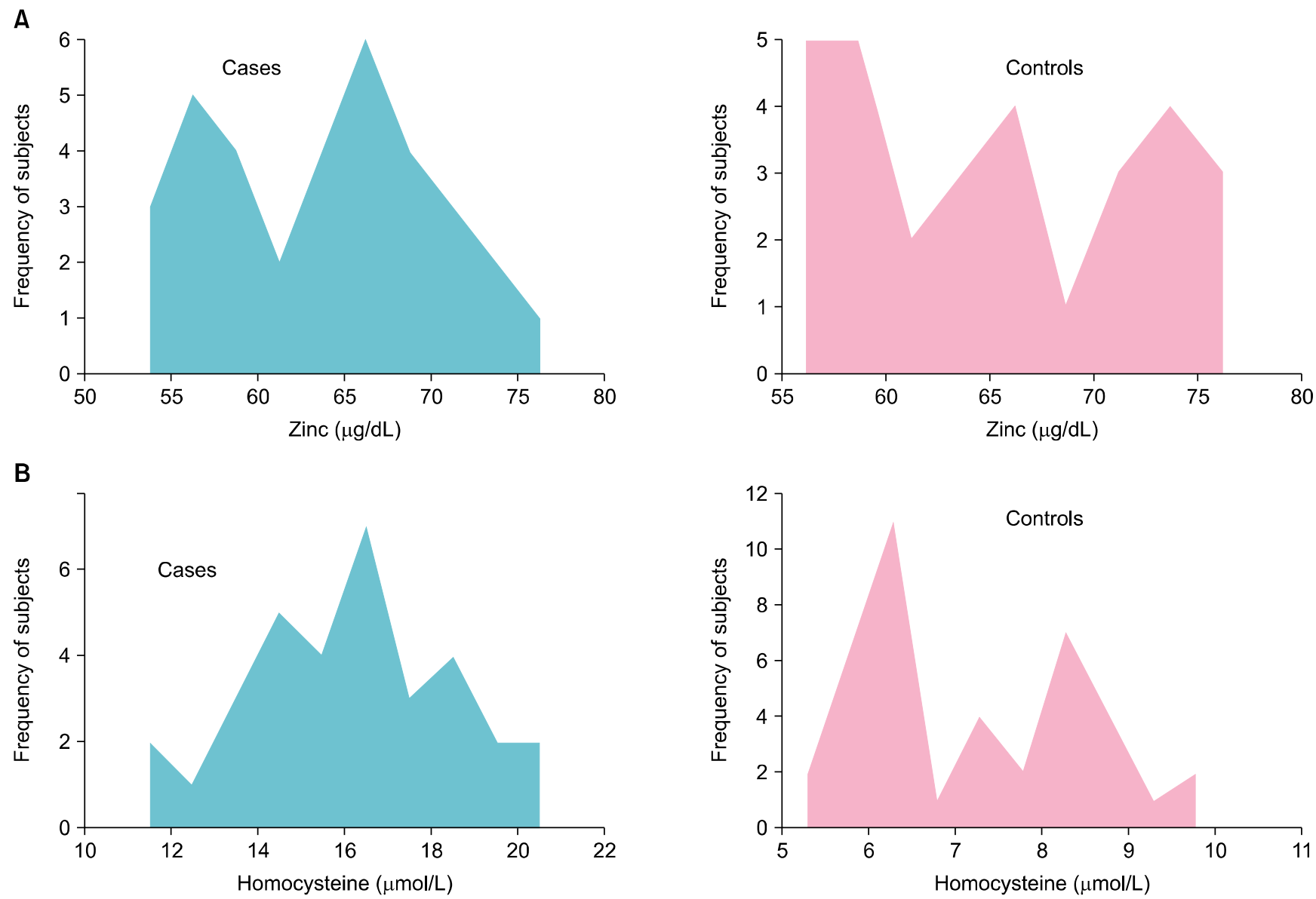

Fig. 1. Mean differences in serum zinc and homocysteine levels in cases and controls. (A) Mean concentration of zinc ( $\mu \mathrm{g} / \mathrm{dL}$ ) was not significantly different in cases as compared to controls $(P>0.05)$. (B) Homocysteine ( $\mu$ mol/L) was significantly higher in cases as compared to controls $(P<0.001)$.

Table 2. Association between serum homocysteine levels and selected predictors

\begin{tabular}{|c|c|c|c|c|c|c|}
\hline \multirow{2}{*}{ Indicator } & \multicolumn{2}{|c|}{ Cases $(n=30)$} & \multicolumn{2}{|c|}{ Controls $(n=30)$} & \multicolumn{2}{|c|}{ Full cohort $(n=60)$} \\
\hline & $\beta$-Coefficient ${ }^{1)}$ & $P$ & $\beta$-Coefficient & $P$ & $\beta$-Coefficient & $P$ \\
\hline Age (years) & 0.21 & 0.09 & 0.18 & 0.40 & 0.04 & 0.78 \\
\hline BMI (1) $\left(\mathrm{kg} / \mathrm{m}^{2}\right)$ & 0.03 & 0.72 & 0.07 & 0.07 & 0.25 & 0.01 \\
\hline BMI (2) $\left(\mathrm{kg} / \mathrm{m}^{2}\right)$ & 0.00 & 0.97 & 0.07 & 0.05 & $0.22^{2)}$ & 0.03 \\
\hline Parity & 0.11 & 0.90 & -0.40 & 0.47 & 3.29 & 0.01 \\
\hline Family history of PE & -0.33 & 0.73 & $-3)$ & - & 4.61 & 0.00 \\
\hline Supplement use & 0.26 & 0.61 & -0.15 & 0.87 & -2.21 & 0.09 \\
\hline Zinc levels $(\mu \mathrm{g} / \mathrm{dL})$ & 0.18 & 0.02 & 0.03 & 0.31 & -0.07 & 0.92 \\
\hline
\end{tabular}

$P$-values $<0.05$ were considered statistically significant.

BMI (1), body mass index before pregnancy; BMI (2), body mass index during pregnancy; PE, pre-eclampsia.

${ }^{1)}$ Indicates the change in Hcy serum levels per unit change in the predicators.

${ }^{2)}$ Controlled for family history and BMI before pregnancy.

${ }^{3)}$ Family history was not introduced to the model as no subject had a family history.

formed. In these models, only age was significantly associated with $\mathrm{Zn}$ levels in the full cohort $(\beta=-0.49$, $P<0.05$ ) (Table 3).

To examine the association between Hcy levels and $\mathrm{Zn}$ status in women with PE and controls, each group, were stratified into two according to indicative cutoffs of serum $\mathrm{Zn}(\mu \mathrm{g} / \mathrm{dL})$. Fig. 2 illustrates mean Hcy levels ( $\mu \mathrm{mol}$ /L) of $\mathrm{Zn}$ deficient and sufficient pregnant women with
$\mathrm{PE}$ and controls. No significant difference were found within the control group $(P>0.05)$, whereas there was a trend toward significance among women with PE $(P=$ 0.05).

Bivariate linear regression analysis was performed to assess the association between blood pressure $(\mathrm{mmHg})$ and serum Hcy levels $(\mu \mathrm{mol} / \mathrm{L})$ in the full cohort. Serum Hcy levels $(\mu \mathrm{mol} / \mathrm{L})$ were entered as independent variables 
Table 3. Association between zinc status and selected predictors

\begin{tabular}{|c|c|c|c|c|c|c|}
\hline \multirow{2}{*}{ Indicator } & \multicolumn{2}{|c|}{ Cases $(n=30)$} & \multicolumn{2}{|c|}{ Controls $(n=30)$} & \multicolumn{2}{|c|}{ Full cohort $(n=60)$} \\
\hline & $\beta$-Coefficient ${ }^{1)}$ & $P$ & $\beta$-Coefficient & $P$ & $\beta$-Coefficient & $P$ \\
\hline Age (years) & -0.49 & 0.08 & -0.34 & 0.32 & -0.49 & 0.03 \\
\hline BMI (1) $\left(\mathrm{kg} / \mathrm{m}^{2}\right)$ & 0.45 & 0.15 & 0.09 & 0.87 & 0.36 & 0.18 \\
\hline BMI (2) $\left(\mathrm{kg} / \mathrm{m}^{2}\right)$ & -0.02 & 0.92 & -0.14 & 0.78 & -0.21 & 0.40 \\
\hline Parity & 2.04 & 0.48 & 1.33 & 0.76 & 0.62 & 0.77 \\
\hline Family history of PE & $5.23^{2)}$ & 0.05 & $-{ }^{3)}$ & - & 2.44 & 0.32 \\
\hline Supplement use & -2.73 & 0.35 & 1.82 & 0.58 & -0.09 & 0.95 \\
\hline
\end{tabular}

$P$-values $<0.05$ were considered statistically significant.

BMI (1), body mass index before pregnancy; BMI (2), body mass index during pregnancy; PE, pre-eclampsia.

${ }^{1)}$ Indicates the change in serum zinc levels per unit change in the predicators.

${ }^{2)}$ Controlled for supplement use.

${ }^{3)}$ Family history was not introduced to the model as no subject had a family history.

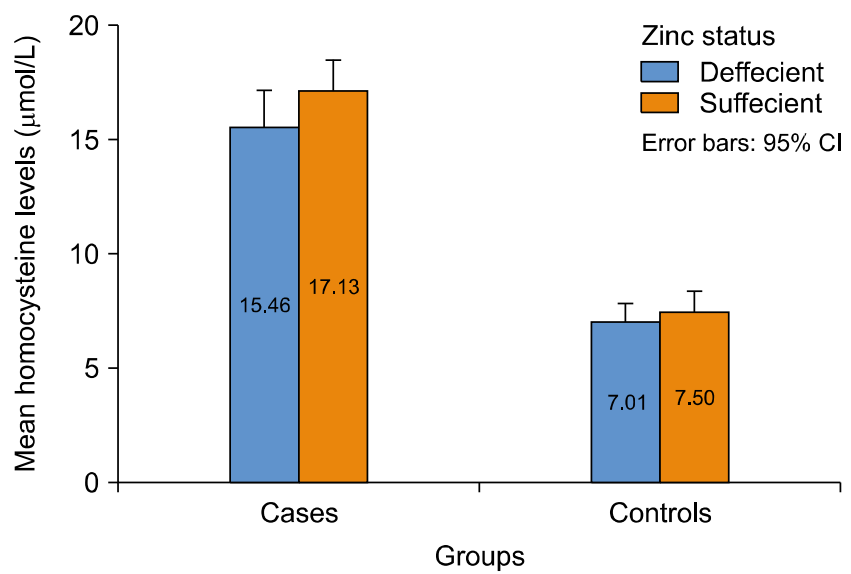

Fig. 2. Homocysteine levels ( $\mu \mathrm{mol} / L$ ) in zinc deficient pregnant women and zinc sufficient pregnant women in both cases and controls. Zinc deficient defined as $\leq 65(\mu \mathrm{g} / \mathrm{dL})$; zinc sufficient defined as $\geq 65$ ( $\mu \mathrm{g} / \mathrm{dL}$ ). No significant difference was found within cases and controls ( $P>0.05)$. Mean $\pm 95 \%$ confidence intervals (CI).

whereas systolic and diastolic blood pressure $(\mathrm{mmHg})$ were entered as dependent variables. An increase of serum Hcy levels of $1 \mu \mathrm{mol} / \mathrm{L}$ was associated with an $3.5 \mathrm{mmHg}$ increase in systolic blood pressure and a $1.8 \mathrm{mmHg}$ increase in diastolic blood pressure $(P<0.05)$. These associations between $\mathrm{Hcy}(\mu \mathrm{mol} / \mathrm{L})$ and systolic and diastolic blood pressure (mmHg) are shown in Fig. 3 and 4, respectively. Logistic regression analyses were performed to examine the associations between H-Hcy and selected predictors. In this model, H-Hcy was entered as the dependent variable and the model was adjusted for blood pressure. When all predictors were entered, only BMI before and during pregnancy was significantly associated with H-Hcy risk (BMI (1): OR=1.27, 95\% CI: 1.12 1.51; BMI (2): $\mathrm{OR}=1.28,95 \%$ CI: 1.13 1.54; $P \leq 0.05)$. Zn levels were inversely associated with increased H-Hcy risk after further adjustment for supplement use. Both systolic and diastolic blood pressure were positively associated with H-Hcy risk (Table 4).

Table 5 shows the risk of selected risk factors on PE in multiple models. Data are presented as OR with 95\% CI

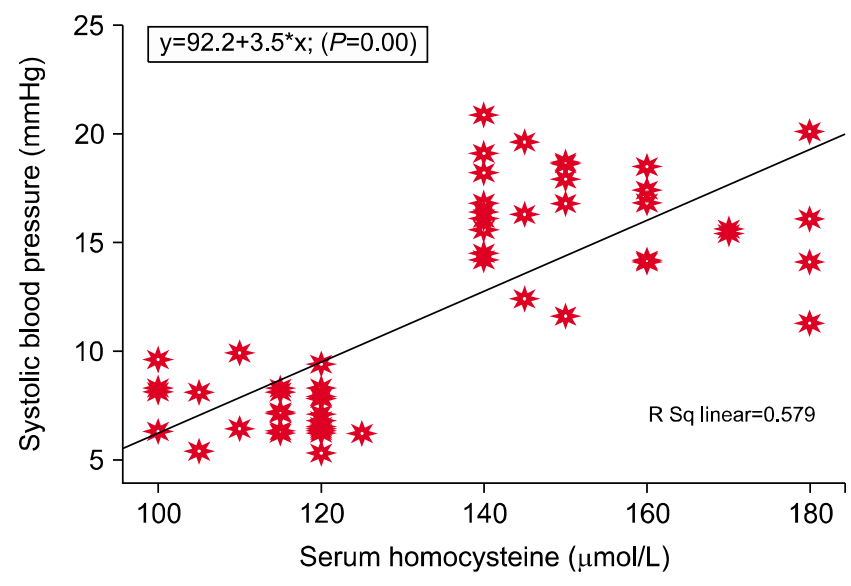

Fig. 3. Predicted systolic blood pressure per unit change of serum homocysteine levels. The straight line represents the linear regression calculations with the corresponding regression equation of the model. Systolic blood pressure $(\mathrm{mmHg})$ was entered as the dependent variables.

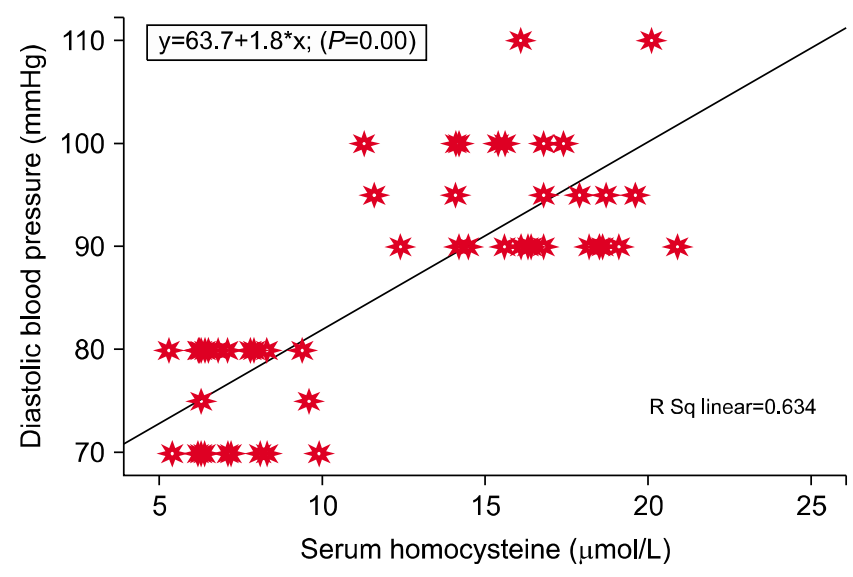

Fig. 4. Predicted diastolic blood pressure per unit change of serum homocysteine levels. The straight line represents the linear regression calculations with the corresponding regression equation of the model. Diastolic blood pressure $(\mathrm{mmHg})$ was entered as the dependent variables.

and $P$-values. All values were adjusted for serum Hcy levels and blood pressure. $\mathrm{Zn}(\mu \mathrm{g} / \mathrm{dL})$ levels were inversely associated with risk of $\mathrm{PE}(\mathrm{OR}=0.84,95 \% \mathrm{CI}$ : $0.71 \sim$ 
Table 4. Association between hyperhomocysteinemia risk and selected predictors ${ }^{1)}$

\begin{tabular}{lcc}
\hline \multicolumn{1}{c}{ Variable } & OR $(95 \% \mathrm{CI})$ & $P$-value \\
\hline Age (years) & $0.93(0.77 \sim 1.14)$ & $\mathrm{NS}$ \\
BMI $(1)\left(\mathrm{kg} / \mathrm{m}^{2}\right)^{2)}$ & $1.27(1.12 \sim 1.51)$ & 0.006 \\
BMI $(2)\left(\mathrm{kg} / \mathrm{m}^{2}\right)^{2)}$ & $1.28(1.13 \sim 1.54)$ & 0.005 \\
Gestational age (weeks) & $0.96(0.83 \sim 1.27)$ & $\mathrm{NS}$ \\
Zinc $(\mu \mathrm{g} / \mathrm{dL})^{3)}$ & $0.87(0.77 \sim 0.99)$ & 0.044 \\
Parity (yes/no $)^{4)}$ & $0.21(0.05 \sim 0.88)$ & 0.03 \\
Supplement use (yes/no $)^{4)}$ & $0.26(0.06 \sim 0.99)$ & 0.04 \\
Family history (yes/no) & $22.47(0.01 \sim 1.44)$ & $\mathrm{NS}$ \\
Systolic blood pressure $(\mathrm{mmHg})$ & $1.14(1.12 \sim 1.18)$ & 0.003 \\
Diastolic blood pressure $(\mathrm{mmHg})$ & $1.21(1.07 \sim 1.34)$ & 0.002 \\
\hline
\end{tabular}

${ }^{1)}$ Model adjusted for blood pressure.

${ }^{2)}$ Controlling for BMI (1) or BMI (2).

${ }^{3)}$ Controlled for supplement use.

${ }^{4)}$ Controlled for age.

BMI (1), body mass index before pregnancy; BMI (2), body mass index during pregnancy; $\mathrm{OR}$, odd ratios; $\mathrm{CI}$, confidence intervals; NS, not significant.

0.98; $P=0.03)$. Supplement use during pregnancy was negatively associated with the risk of $\mathrm{PE}(\mathrm{OR}=0.21,95 \%$ CI: 0.05 0.81; $P=0.02$ ) after adjusting for family history. Parity was associated with an increased odds of PE (OR $=7.42$, 95\% CI: 1.34 41.06; $P=0.02$ ). Furthermore, increased BMI during pregnancy was positively associated with risk of $\mathrm{PE}(\mathrm{OR}=1.14,95 \% \mathrm{CI}: 1.12 \sim 1.33 ; P=0.02)$. Although serum Hcy levels ( $\mu \mathrm{mol} / \mathrm{L}$ ) were significantly higher among women with PE than controls, these differences did not affect the odds of PE reaching the desired significance level $(P>0.05)$ in the risk analysis.

\section{DISCUSSION}

PE is a public health risk in both developed and developing countries, and accounts for most maternal and perinatal morbidity and mortality globally (Yelikar et al., 2016). In the present study, there were no significant differences between PE pregnant women and healthy pregnant women in terms of age, gestational age, and BMI before pregnancy. However, BMI during pregnancy was significantly higher in pregnant women with $\mathrm{PE}$ than that in healthy pregnant women. This difference could be related to increased water retention (edema) and protein in urine (proteinuria) in PE pregnant women, which is associated with swelling and an abnormal increment in weight (Kanagal et al., 2014).

In our study, serum Hcy was higher in women with PE than in controls $(16.35 \mu \mathrm{mol} / \mathrm{L}$ vs $7.25 \mu \mathrm{mol} / \mathrm{L}$, respectively; $P<0.001)$. A similar trend was found by Makedos et al. (2007) in which mean Hcy levels were $11.11 \mu \mathrm{mol} /$ $\mathrm{L}$ in pregnant women with PE compared with $6.4 \mu \mathrm{mol} / \mathrm{L}$ in healthy pregnant women. Furthermore, Harma et al. (2005) investigated the association between levels of Hcy,
Table 5. Risk of pre-eclampsia and selected predictors of pre-eclampsia ${ }^{1)}$

\begin{tabular}{lcc}
\hline \multicolumn{1}{c}{ Variable } & OR $(95 \% \mathrm{CI})$ & $P$-value \\
\hline Age (years) & $0.99(0.87 \sim 1.12)$ & $\mathrm{NS}$ \\
BMI $(1)\left(\mathrm{kg} / \mathrm{m}^{2}\right)$ & $1.20(1.15 \sim 1.44)$ & 0.02 \\
BMI (2) $\left(\mathrm{kg} / \mathrm{m}^{2}\right)$ & $1.14(1.12 \sim 1.33)$ & 0.02 \\
Gestational age (weeks) & $1.04(0.96 \sim 1.25)$ & $\mathrm{NS}$ \\
Zinc ( $\mu \mathrm{g} / \mathrm{dL})$ & $0.84(0.71 \sim 0.98)$ & 0.03 \\
Homocysteine ( $\mu \mathrm{mol} / \mathrm{L})$ & $13.64(0.90 \sim 23.03)$ & $\mathrm{NS}$ \\
Parity (yes/no) & $7.42(1.34 \sim 41.06)$ & 0.02 \\
Supplement use (yes/no) & $0.21(0.05 \sim 0.81)$ & 0.02 \\
Family history (yes/no $)^{2)}$ & $22.42(0.08 \sim 1.46)$ & $\mathrm{NS}$ \\
Systolic blood pressure $(\mathrm{mmHg})$ & $1.57(0.03 \sim 4.47)$ & $\mathrm{NS}$ \\
Diastolic blood pressure $(\mathrm{mmHg})$ & $0.18(0.06 \sim 0.99)$ & $\mathrm{NS}$ \\
\hline
\end{tabular}

${ }^{1)}$ Model adjusted for homocysteines and blood pressure.

${ }^{2)}$ Controlled for family history.

BMI (1), body mass index before pregnancy; BMI (2), body mass index during pregnancy; NS, not significant.

Zn, copper, and PE using a case control design. The study included 24 pregnant women with PE and 44 normotensive pregnant controls. The authors found that Hcy levels were higher in pregnant women with PE than in normotensive controls $(16.39 \pm 9.32 \mathrm{nmol} / \mathrm{mL}$ vs $9.45 \pm 3.64$ $\mathrm{nmol} / \mathrm{mL}$, respectively). Another study conducted in Iran by Hasanzadeh et al. (2008) showed that women with PE have higher Hcy levels than normal pregnant women.

However, results from studies investigating the association between serum Hcy and PE are contradictory. For example, a case control study conducted by Shilpa et al. (2017) demonstrated significantly lower serum Hcy level in PE patients $(8.90 \pm 4.33 \mu \mathrm{mol} / \mathrm{L})$ compared with normal non-pregnant women $(13.31 \pm 5.81 \mu \mathrm{mol} / \mathrm{L})$. However, mean serum Hcy levels of the PE patients $(8.90 \pm 4.33$ $\mu \mathrm{mol} / \mathrm{L})$ were not significantly higher than those of normal pregnant women $(7.52 \pm 2.25 \mu \mathrm{mol} / \mathrm{L})$. The authors indicated that this insignificant minor difference in serum Hcy levels may be due to PE patients receiving folic acid supplementation during their first trimester of gestation. In our study, serum Hcy levels did not significantly differ between supplement users and non-supplement users in women with PE and controls; however, the likelihood of $\mathrm{H}$-Hcy was significantly lower in supplement users vs non-users (OR=0.26,95\% CI: 0.06 0.99; $P=0.04)$.

Our study demonstrated the effect of serum Hcy levels on the severity of the disease. There was a strong association between the severity of hypertension and levels of serum Hcy. An increase in serum Hcy of $1 \mu \mathrm{mol} / \mathrm{L}$ was associated with a $3.5 \mathrm{mmHg}$ increase in systolic blood pressure and $1.8 \mathrm{mmHg}$ increase in diastolic blood pressure $(P<0.05)$. Our results are consistent with a previous study that showed higher maternal serum Hcy levels in women with severe PE compared with women with mild PE (6.38 \pm 0.3 vs. $14.05 \pm 1.43$, respectively) (Khosrowbeygi and Ahmadvand, 2011). A recent case control 
study involving 51 PE pregnant women examined the association between serum Hcy levels and the severity of PE. The authors reported significantly higher serum Hcy levels in pregnant women with severe PE compared with women with non-severe disease $(10.67 \pm 1.49$ vs $7.76 \pm$ 1.88 , respectively; $P<0.05$ ) (Shahbazian et al., 2016). Similarly, in a 2-year prospective cohort study, the association between Hcy levels and severity of hypertension and complications of PE and eclampsia was confirmed (Maru et al., 2016).

Many studies have indicated that H-Hcy is a well-recognized risk factor for cardiovascular diseases. Accordingly, many studies have addressed the possible causal relationship between H-Hcy and adverse pregnancy outcomes (Dodds et al., 2008). Plasma Hcy concentrations normally decrease during pregnancy via an unknown mechanism, which may involve normal increases in glomerular filtration rates that accompany pregnancy, increases in plasma volume and associated hemodilution, and increased uptake of Hcy by the fetus (López-Quesada et al., 2003; Harma et al., 2005). Elevated levels of Hcy have been postulated to produce oxidative stress and endothelial dysfunction, which are associated with $\mathrm{PE}$ (Mignini et al., 2005). However, there are contradictory data on the potential of Hcy as a marker for subsequent PE. Some studies suggest that H-Hcy in early pregnancy can increase the risk of developing PE (Cotter et al., 2003; López-Quesada et al., 2003; Dodds et al., 2008). However, Zeeman et al. (2003) showed that Hcy concentrations do not significantly differ between women with PE and normal pregnant women, and that maternal Hcy concentrations cannot be used as a predicator for PE (Zeeman et al., 2003). In this study, serum Hcy levels $(\mu \mathrm{mol} / \mathrm{L})$ were significantly higher among pregnant women with $\mathrm{PE}$, but their differences did not affect the odds of PE reaching the desired significance level $(\mathrm{OR}=13.64,95 \%$ CI: $0.90 \sim 23.03 ; P>0.05)$ in the risk analysis. In the current study, the small sample size or other unconsidered confounders may have masked the association. In addition, pregnancy is associated with a decrease in serum Zn concentration (Kumru et al., 2003); this alteration may include physiological adjustment to pregnancy, a response to hormone changes, hemodilution, or combination of these factors (Tamura et al., 2000).

The demands for micronutrients during pregnancy often cannot be met in low-income countries due to poor diets (Gernand et al., 2016). For example in South Asia, the prevalence of $\mathrm{Zn}$ deficiencies ranges from $15 \sim 74 \%$ (Gernand et al., 2016). In our study, approximately $50 \%$ of the participants were $\mathrm{Zn}$ deficient. Age, parity, socioeconomic status and other factors might influence nutrition during gestation (Gernand et al., 2016). We showed that $\mathrm{Zn}$ was associated with age in the full sample but not in women with PE or controls. Indeed, a one-year increase in age was significantly associated with a 0.49 $\mu \mathrm{g} / \mathrm{dL}$ decrease in serum $\mathrm{Zn}$ levels $(P=0.03)$; however, there was no association between serum $\mathrm{Zn}$ levels and other predictors in women with $\mathrm{PE}$ and controls $(P>$ 0.05). Unfortunately, no information was available about dietary intake of $\mathrm{Zn}$ in our sample population, which is the main factor that determines $\mathrm{Zn}$ status. In this context, many previous studies have explored the relationship between changes in serum $\mathrm{Zn}$ levels in pregnant women and PE. However, results from these studies are conflicting. Some studies found lower serum $\mathrm{Zn}$ is significantly higher in pregnant women with PE compared with healthy pregnant women (Ilhan et al., 2002; Farzin and Sajadi, 2012; Sarwar et al., 2013). However, other studies showed that serum $\mathrm{Zn}$ levels are higher in pregnant women with PE than in healthy pregnant women (Mahomed et al., 2000; Harma et al., 2005; Katz et al., 2012). Our data did not show any significant differences in $\mathrm{Zn}$ levels between pregnant women with $\mathrm{PE}$ and controls $(63.71 \pm 1.24 \mu \mathrm{g} / \mathrm{dL}$ and $65.37 \pm 1.27 \mu \mathrm{g} / \mathrm{dL}$, respectively; $P>0.05)$.

Our findings are in agreement with Golmohammadlou et al. (2008) and Vigeh et al. (2006), who showed that the serum $\mathrm{Zn}$ concentrations do not significantly differ between pregnant women with PE and healthy pregnant women. These results may be explained by the negative impact of iron on $\mathrm{Zn}$ absorption and status that could further exacerbate $\mathrm{Zn}$ status (Lönnerdal, 2000). In our study, approximately $63 \%$ of full cohort used supplements (calcium, iron, or multivitamins), which could explain why both pregnant women with PE and controls were at borderline of the normal range of the $\mathrm{Zn}$ cutoff level (i.e., $65.00 \mu \mathrm{g} / \mathrm{dL}$ ) (Álvarez et al., 2007). However, elevated $\mathrm{Zn}$ in blood was significantly associated with $\mathrm{H}-\mathrm{Hcy}$ in PE, and Hcy concentrations were positively correlated with plasma $\mathrm{Zn}$ concentrations in women with PE $(r=0.588 ; P=0.003)$ but not healthy controls (Harma et al., 2005). In pregnant women with PE, a one unit increase in serum $\mathrm{Zn}$ was significantly associated with 0.18 $\mu \mathrm{mol} / \mathrm{L}$ decrease in serum Hcy levels $(P=0.02)$; however, there was no association between serum $\mathrm{Zn}$ and serum Hcy in controls or the full cohort $(P>0.05)$.

Limitations of the present study include the small sample and a lack of information about dietary $\mathrm{Zn}$ intake, both of which may mask the influence of $\mathrm{Zn}$ status on Hcy levels and PE risk in pregnant women. A greater prospective and more population based studies are needed to further explore the association between elevated Hcy, low vitamins and $\mathrm{Zn}$ status, and the risk of PE. Overall, early management of H-Hcy and the associated risk factors may be effective in decreasing the incidence of PE in our population. 


\section{ACKNOWLEDGEMENTS}

This work was supported by the Faculty of Graduate Studies at the University of Jordan. We wish to especially thank the Obstetrics and Gynecology Department at Al-Bashier Hospital and Al-Husain Medical Center for recruiting and coordinating subjects.

\section{AUTHOR DISCLOSURE STATEMENT}

The authors declare no conflict of interest.

\section{REFERENCES}

Akhtar S. Zinc status in South Asian populations - an update. J Health Popul Nutr. 2013. 31:139-149.

Akinloye O, Oyewale OJ, Oguntibeju OO. Evaluation of trace elements in pregnant women with pre-eclampsia. Afr J Biotechnol. 2010. 9:5196-5202.

Álvarez SI, Castañón SG, Ruata MLC, Aragüés EF, Pilar Bocos Terraz, Irazabal YG, et al. Updating of normal levels of copper, zinc and selenium in serum of pregnant women. J Trace Elem Med Biol. 2007. 21:49-52.

Cotter AM, Molloy AM, Scott JM, Daly SF. Elevated plasma homocysteine in early pregnancy: a risk factor for the development of nonsevere preeclampsia. Am J Obstet Gynecol. 2003. 189:391-396.

Dodds L, Fell DB, Dooley KC, Armson BA, Allen AC, Nassar BA, et al. Effect of homocysteine concentration in early pregnancy on gestational hypertensive disorders and other pregnancy outcomes. Clin Chem. 2008. 54:326-334.

Farzin L, Sajadi F. Comparison of serum trace element levels in patients with or without pre-eclampsia. J Res Med Sci. 2012. 17:938-941.

Gernand AD, Schulze KJ, Stewart CP, West KP Jr, Christian P. Micronutrient deficiencies in pregnancy worldwide: health effects and prevention. Nat Rev Endocrinol. 2016. 12:274-289.

Ghulmiyyah L, Sibai B. Maternal mortality from preeclampsia/ eclampsia. Semin Perinatol. 2012. 36:56-59.

Golmohammadlou S, Amirabi A, Yazdian M, Pashapour N. Evaluation of serum calcium, magnesium, copper, and zinc levels in women with pre-eclampsia. Iran J Med Sci. 2008. 33:231234.

Harma M, Harma M, Kocyigit A. Correlation between maternal plasma homocysteine and zinc levels in preeclamptic women. Biol Trace Elem Res. 2005. 104:97-105.

Hasanzadeh M, Ayatollahi H, Farzadnia M, Ayati S, Khoob MK. Elevated plasma total homocysteine in preeclampsia. Saudi Med J. 2008. 29:875-878.

Ilhan N, Ilhan N, Simsek M. The changes of trace elements, malondialdehyde levels and superoxide dismutase activities in pregnancy with or without preeclampsia. Clin Biochem. 2002. 35:393-397.

Jing M, Rech L, Wu Y, Goltz D, Taylor CG, House JD. Effects of zinc deficiency and zinc supplementation on homocysteine levels and related enzyme expression in rats. J Trace Elem Med Biol. 2015. 30:77-82.

Kanagal DV, Rajesh A, Rao K, Shetty H, Shetty PK, Ullal H. Zinc and copper levels in preeclampsia: a study from coastal South India. Int J Reprod Contracept Obstet Gynecol. 2014. 3:370373.
Katz O, Paz-Tal O, Lazer T, Aricha-Tamir B, Mazor M, Wiznitzer A, et al. Severe pre-eclampsia is associated with abnormal trace elements concentrations in maternal and fetal blood. J Matern Fetal Neonatal Med. 2012. 25:1127-1130.

Khosrowbeygi A, Ahmadvand H. Circulating levels of homocysteine in preeclamptic women. Bangladesh Med Res Counc Bull. 2011. 37:106-109.

Kumru S, Aydin S, Simsek M, Sahin K, Yaman M, Ay G. Comparison of serum copper, zinc, calcium, and magnesium levels in preeclamptic and healthy pregnant women. Biol Trace Elem Res. 2003. 94:105-112.

Lönnerdal B. Dietary factors influencing zinc absorption. J Nutr. 2000. 130:1378S-1383S.

López-Quesada E, Vilaseca MA, Lailla JM. Plasma total homocysteine in uncomplicated pregnancy and in preeclampsia. Eur J Obstet Gynecol Reprod Biol. 2003. 108:45-49.

Mahomed K, Williams MA, Woelk GB, Mudzamiri S, Madzime S, King IB, et al. Leukocyte selenium, zinc, and copper concentrations in preeclamptic and normotensive pregnant women. Biol Trace Elem Res. 2000. 75:107-118.

Makedos G, Papanicolaou A, Hitoglou A, Kalogiannidis I, Makedos A, Vrazioti V, et al. Homocysteine, folic acid and B12 serum levels in pregnancy complicated with preeclampsia. Arch Gynecol Obstet. 2007. 275:121-124.

Maru L, Verma M, Jinsiwale N. Homocysteine as predictive marker for pregnancy-induced hypertension - a comparative study of homocysteine levels in normal versus patients of PIH and its complications. J Obstet Gynaecol India. 2016. 66:167-171.

Mignini LE, Latthe PM, Villar J, Kilby MD, Carroli G, Khan KS. Mapping the theories of preeclampsia: the role of homocysteine. Obstet Gynecol. 2005. 105:411-425.

Rafeeinia A, Tabandeh A, Khajeniazi S, Marjani AJ. Serum copper, zinc and lipid peroxidation in pregnant women with preeclampsia in gorgan. Open Biochem J. 2014. 8:83-88.

Roberts JM, Balk JL, Bodnar LM, Belizán JM, Bergel E, Martinez A. Nutrient involvement in preeclampsia. J Nutr. 2003. 133: 1684S-1692S.

Sarwar MS, Ahmed S, Ullah MS, Kabir H, Rahman GK, Hasnat A, et al. Comparative study of serum zinc, copper, manganese, and iron in preeclamptic pregnant women. Biol Trace Elem Res. 2013. 154:14-20.

Shahbazian N, Jafari RM, Haghnia S. The evaluation of serum homocysteine, folic acid, and vitamin B12 in patients complicated with preeclampsia. Electron Physician. 2016. 8:3057-3061.

Shilpa AV, Zubaida PA, Rajalekshmi G. Changes in homocysteine levels during normal pregnancy and preeclampsia and its relation with oxidative stress. Int J Res Med Sci. 2017. 5:330-334.

Tamura T, Goldenberg RL, Johnston KE, DuBard M. Maternal plasma zinc concentrations and pregnancy outcome. Am J Clin Nutr. 2000. 71:109-113.

Tug N, Celik H, Cikim G, Ozcelik O, Ayar A. The correlation between plasma homocysteine and malondialdehyde levels in preeclampsia. Neuro Endocrinol Lett. 2003. 24:445-448.

Vafaei H, Dalili M, Hashemi SA. Serum concentration of calcium, magnesium and zinc in normotensive versus preeclampsia pregnant women: a descriptive study in women of Kerman province of Iran. Iran J Reprod Med. 2015. 13:23-26.

Vigeh M, Yokoyama K, Ramezanzadeh F, Dahaghin M, Sakai T, Morita Y, et al. Lead and other trace metals in preeclampsia: a case-control study in Tehran, Iran. Environ Res. 2006. 100: 268-275.

WHO. Managing complications in pregnancy and childbirth: a guide for midwives and doctors. World Health Organization, Geneva, Switzerland. 2017 [cited 2019 Sep 18]. Available from: https://apps.who.int/iris/bitstream/handle/10665/255760/ 9789241565493-eng.pdf

Yelikar KA, Deshpande SS, Kulkarni ML. Association of maternal 
serum homocysteine level with severity of preeclampsia: a case control study. 2016. 5:2713-2717.

Zeeman GG, Alexander JM, McIntire DD, Devaraj S, Leveno KJ.
Homocysteine plasma concentration levels for the prediction of preeclampsia in women with chronic hypertension. Am J Obstet Gynecol. 2003. 189:574-576. 